\title{
"Mosaic Pattern" Foreign Bodies in Computed Tomography of the Head: A Specific Sign to Detect Tempered Glass-Related Head Injury
}

\author{
Mohd. Faheem $1,(10)$ \\ Raj Kumar $^{2} \quad$ Manish Jaiswal ${ }^{3}$

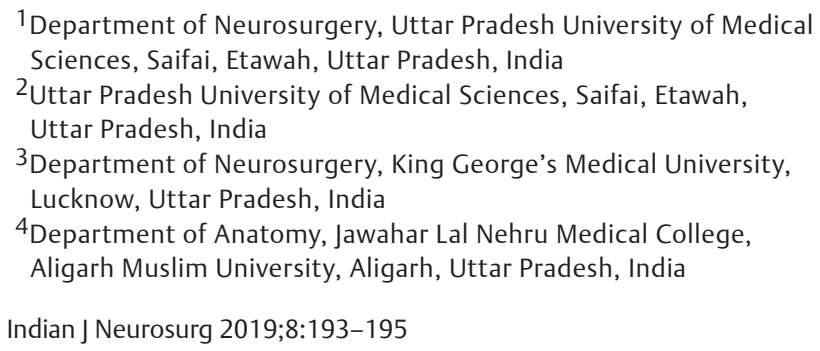

Mohammad Ahmed Ansari ${ }^{1}$

Noor us Saba ${ }^{4}$

\author{
Address for correspondence Mohd. Faheem, MBBS, MS, MCh, \\ Department of Neurosurgery, Uttar Pradesh University of \\ Medical Sciences, Saifai, Etawah 206130, Uttar Pradesh, India \\ (e-mail: faheemjnmc@gmail.com).
}

\begin{abstract}
A 40-year-old male patient presented to us with a history of road traffic accident, followed by loss of consciousness and vomiting. Noncontrast computed tomography (NCCT) scan revealed frontal bone defect along with multiple "mosaic pattern" homogenously hyperdense foreign bodies of nearly similar size in the right frontoparietal region within the extradural space. Debridement craniectomy was done, and multiple pieces of glass were retrieved. The extremely unique radiologic finding and probably first in the English literature prompted us to report this interesting case. Unfortunate-

Keywords

- mosaic

- penetrating

- tempered glass ly, the patient died of myocardial infarction 3 days after the injury. Further, this new "mosaic pattern" radiologic finding on NCCT of the head may help in determining the mechanism of injury due to tempered glass-related head trauma. Window glass may not be always safe as it is thought by commuters.
\end{abstract}

\section{Introduction}

Foreign objects are often embedded into the skull during the penetrating head trauma. Most of the intracranial foreign bodies occur around the orbit, frontal sinus, and the nasal areas due to the thinness of bone at such places. Numerous type of foreign bodies such as wood, pellet, stone, umbrella tip, pencil, nail, keys, and knitting needles have been reported in the literature. ${ }^{1,2}$ Rarely, glass as a foreign body has also been described through temporal and parietal bone, but "mosaic pattern" of tempered glass on computed tomography (CT) of the head is completely a new finding that can be used in future to determine the mechanism of injury due to tempered glass-related injury.,4

In this report, we present a rare case of tempered glassrelated injury, unique radiologic finding, possible complications, and their management.

received

March 8, 2019

accepted

May 1, 2019

published online

November 15, 2019

\section{Summary}

A 40-year-old male patient presented to the emergency department (ED) after he sustained a head injury due to overturning of a passenger bus following road traffic accident (RTA). He sustained injury to right side of the cranium as he was sitting adjacent to the window. There was a history of bleeding from the scalp wound, loss of consciousness, vomiting, and multiple glass pieces in the wound. The patient had a previous history of ischemic heart disease for which cardiac stenting was performed 5 years back. As per history, he was on irregular follow-up in cardiac outpatient department (OPD) and was on statins and antiplatelet drugs. He was also advised cardiac angiography 1 year back, but it could not be done due to financial constraints.

(C)2019 Neurological Surgeons'

Society of India
License terms

(요 (1) $\odot \circledast$
$10.1055 / \mathrm{s}-0039-1700302$

ISSN 2277-954X. 
Upon arrival at the ED, the patient was managed as per standard Advanced Trauma Life Support (ATLS) protocol. His vitals were stable, and Glasgow coma scale (GCS) score was E1V2M5 $(8 / 15)$. There was a wound of size $10 \times 4 \mathrm{~cm}$ in the right frontoparietal region along with multiple pieces of glass within the wound. The patient was immediately intubated, and pressure bandage was applied at the wound. There was no long bone fracture or any other associated injury. Rest of the examination including musculoskeletal, respiratory, and abdominal were unremarkable.

Noncontrast CT (NCCT) of the head was performed to evaluate the extent of brain injury. CT revealed a right frontal bone defect of $3 \times 4 \mathrm{~cm}$ in size along with multiple similar-sized hyperdense foreign bodies (mosaic pattern) within this compact area in the right frontoparietal region within the extradural space ( - Fig. 1C, D). There was a local compression of the brain parenchyma.

\section{Treatment}

Debridement craniectomy was done, and multiple pieces of glasses were retrieved from the wound (-Fig. 1B). The glass pieces were found within the extradural space only. The dura was thoroughly inspected to look for any breach due to the glass fragments. Wound was thoroughly cleaned with normal saline and antiseptic solution. Wound was closed using nylon suture, and broad-spectrum antibiotics were administered to prevent the wound infection.

\section{Outcome and Follow-up}

The patient's GCS improved to $11 / 15$ and $13 / 15$ on first and second postoperative days, respectively. He continued on statins, but antiplatelets were stopped before surgery keeping strict watch on bleeding time (BT), clotting time (CT), and international normalized ratio (INR).
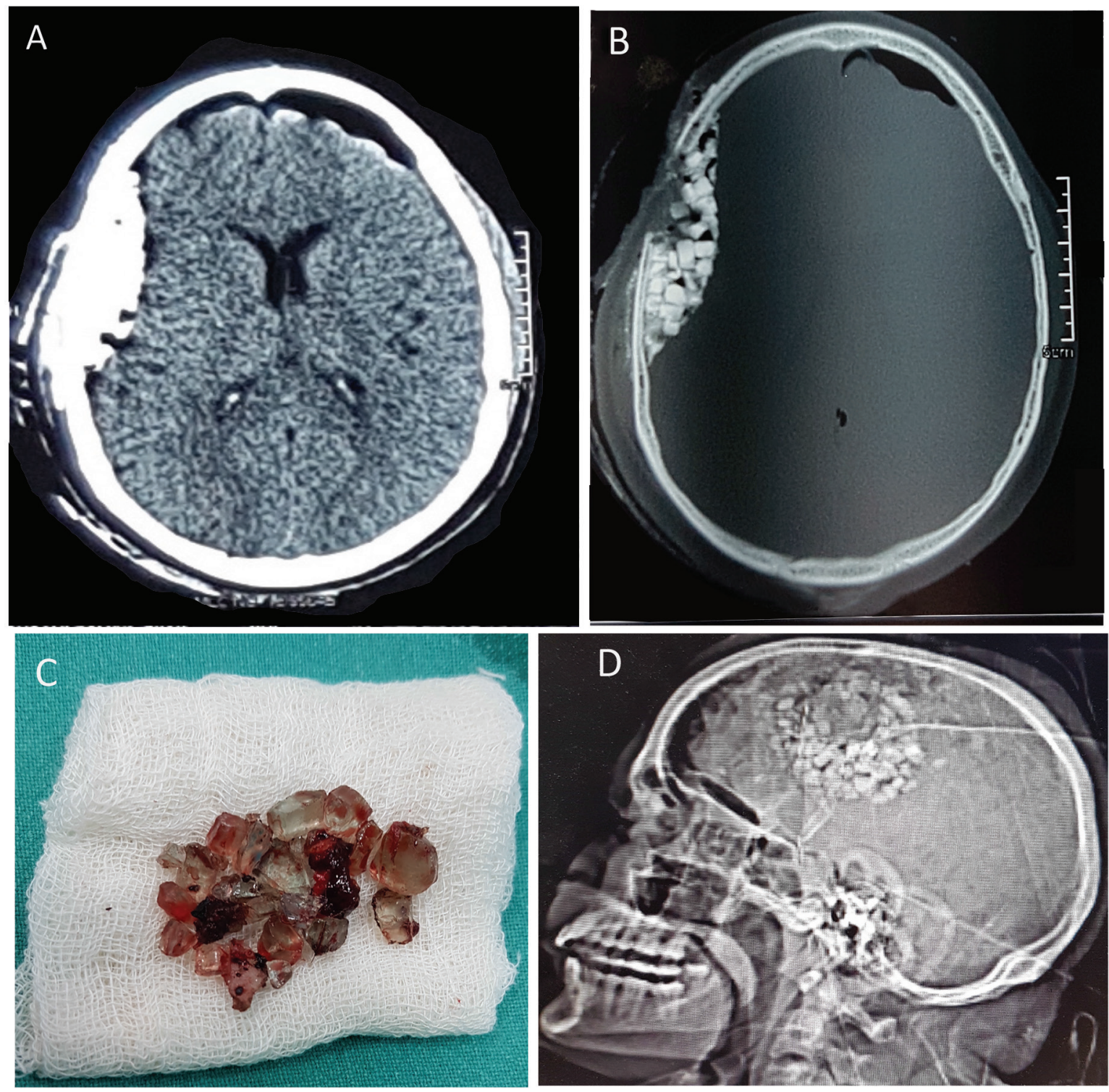

Fig. 1 (A, B) NCCT head along with bone window showing mosaic pattern within the extradural space. (C) Multiple pieces of glass which were retrieved. (D) Skull radiograph showing multiple radio-opaque foreign bodies in fronto-parietal region. 
The parameters remained within normal limits in postoperative period. NCCT of the head was planned at the time of discharge, but unfortunately the patient died of massive myocardial infarction on fourth postoperative day.

\section{Discussion}

Craniocerebral perforating injuries have been reported by a variety of objects such as nails, metal, keys, pencil, gunshot injuries, chopstick, stone, pellet, wood, and power drill, but most commonly they are caused by knife injury. ${ }^{1,25-7}$ As per the literature, intracranial penetration by foreign bodies occur most commonly through the orbit, nose, or frontal sinus. ${ }^{4,89}$ Rarely, penetrating injuries can occur due to glass of an automobile window. Moreover, penetrating injury due to multiple pieces of tempered glass in an extremely hard frontal bone has not been reported previously.

Automobile glass is mainly of two types: toughened or tempered glass and laminated glass. Toughened or tempered glass is used in an automobile window and rear windscreen whereas laminated glasses are present in front windscreen of an automobile. The laminated glass is a type of glass that holds together when shattered. It is held in place by an interlayer, typically of polyvinyl butyral or ethylene vinyl acetate. In the event of breaking, the interlayer keeps the layer of glass bonded and its high strength prevents the glass from breaking into pieces. Tempered glass breaks into multiple pieces in the event of an accident. In our case, the patient was injured due to multiple pieces of tempered glass. ${ }^{10}$

Skull radiograph may also be helpful, especially in peripheral centers, which may reveal multiple radiopaque foreign bodies. NCCT of the head shows better delineation in cases of suspected glass injury. Glass appears hyperdense on CT, and "mosaic pattern" appearance of tempered glass can be used as a specific sign of tempered glass-related head injuries. MRI may also be required where relationship of foreign body to the brainstem, blood vessels, and any other vital structure is required. ${ }^{3}$

The common complications after penetrating head injury includes infection and vascular complications. Infective complication may be in the form of brain abscess and meningitis. Vascular complications that are encountered include pseudoaneurysm, vasospasm, vascular occlusion, arteriovenous fistula, and venous thrombosis. ${ }^{11}$ Cerebral angiography can also be done to rule out these vascular complications. ${ }^{12}$ Glass wound can also result in death due to exsanguination and air embolism. ${ }^{8}$ The recovery can take months or years, and in survivors, residual neurologic deficit in the form of limb weakness, behavioral abnormalities, etc. persists. ${ }^{13}$

\section{Conclusion}

This report suggests that a thorough knowledge of the modes and mechanisms of injury is required to have a high degree of suspicion for penetrating head injury. Glass window (tempered) are not safe and not devoid of penetrating injuries to the head. Moreover, "mosaic pattern" on CT of the head may be regarded as a quite useful sign to detect tempered glass-related head injuries in future.

\section{Financial Support \\ None.}

\section{Conflicts of Interest}

None.

\section{References}

1 Carothers A. Orbitofacial wounds and cerebral artery injuries caused by umbrella tips. JAMA 1978;239(12):1151-1152

2 Jooma R, Bradshaw JR, Coakham HB. Computed tomography in penetrating cranial injury by a wooden foreign body. Surg Neurol 1984;21(3):236-238

3 Sharma A, Pandey A, Diyora B, Shah S, Sayal P, Dwivedi N. Intracranial penetration of a glass piece through the temporal region in civilian head injury. J Trauma 2006;60(4):891-893

4 Nakagawa A, Su CC, Yamashita Y, Endo T, Shirane R. [A temporal head injury involving intracranial penetration by glass] [Japanese]. No Shinkei Geka 2002;30(5):529-533

5 Fathalla $\mathrm{H}$, Ashry A, El-Fiki A. Managing military penetrating brain injuries in the war zone: lessons learned. Neurosurg Focus 2018;45(6):E6

6 Luo W, Liu H, Hao S, Zhang Y, Li J, Liu B. Penetrating brain injury caused by nail guns: two case reports and a review of the literature. Brain Inj 2012;26(13-14):1756-1762

7 Woodall MN, Alleyne CH Jr. Nail-gun head trauma: a comprehensive review of the literature. J Trauma Acute Care Surg 2012;73(4):993-996

8 Srivastava C, Sunil SK, Ojha BK. Intracranial penetrating glass injury: a case report. Indian Journal of Neurotrauma 2012;9:64-66

9 Liau LM, Bergsneider M, Becker DP, Pathology and Pathophysiology of Head Injury. In: Youmans JR, ed. Neurological Surgery. 4th ed, Vol 3. Philadelphia, PA: W.B. Saunders; 1996 1549-1594

10 llen TJ, Cox AR, Barton S, et al. Breaking of flat glass-part 4. Size and distribution of fragments from vehicle windscreens. Forensic Sci Int 1998;93:209-218

11 du Trevou MD, van Dellen JR. Penetrating stab wounds to the brain: the timing of angiography in patients presenting with the weapon already removed. Neurosurgery 1992;31(5):905911, discussion 911-912

12 Kieck CF, de Villiers JC. Vascular lesions due to transcranial stab wounds. J Neurosurg 1984;60(1):42-46

13 Cristofori I, Pal S, Zhong W, Gordon B, Krueger F, Grafman J. The lonely brain: evidence from studying patients with penetrating brain injury. Soc Neurosci 2018 (e-pub ahead of print) doi: 10.1080/17470919.2018.1553798 